\title{
Learning Externalities and Economic Growth
}

\author{
Alejandro M. Rodriguez*
}

August 2004

*I would like to thank Profesor Robert E. Lucas for motivating me to write this paper and for his helpful comments. Profesor Alvarez also provided some useful insights. Please send comments to amr@cema.edu.ar. All errors are mine. 


\begin{abstract}
It is a well known fact that not all countries develop at the same time. The industrial revolution began over 200 years ago in England and has been spreading over the world ever since. In their paper Barriers to Riches, Parente and Prescott notice that countries that enter the industrial stage later on grow faster than what the early starters did. I present a simple model with learning externalities that generates this kind of behavior. I follow Lucas (1998) and solve the optimization problem of the representative agent under the assumption that the external effect is given by the world leader's human capital.
\end{abstract}




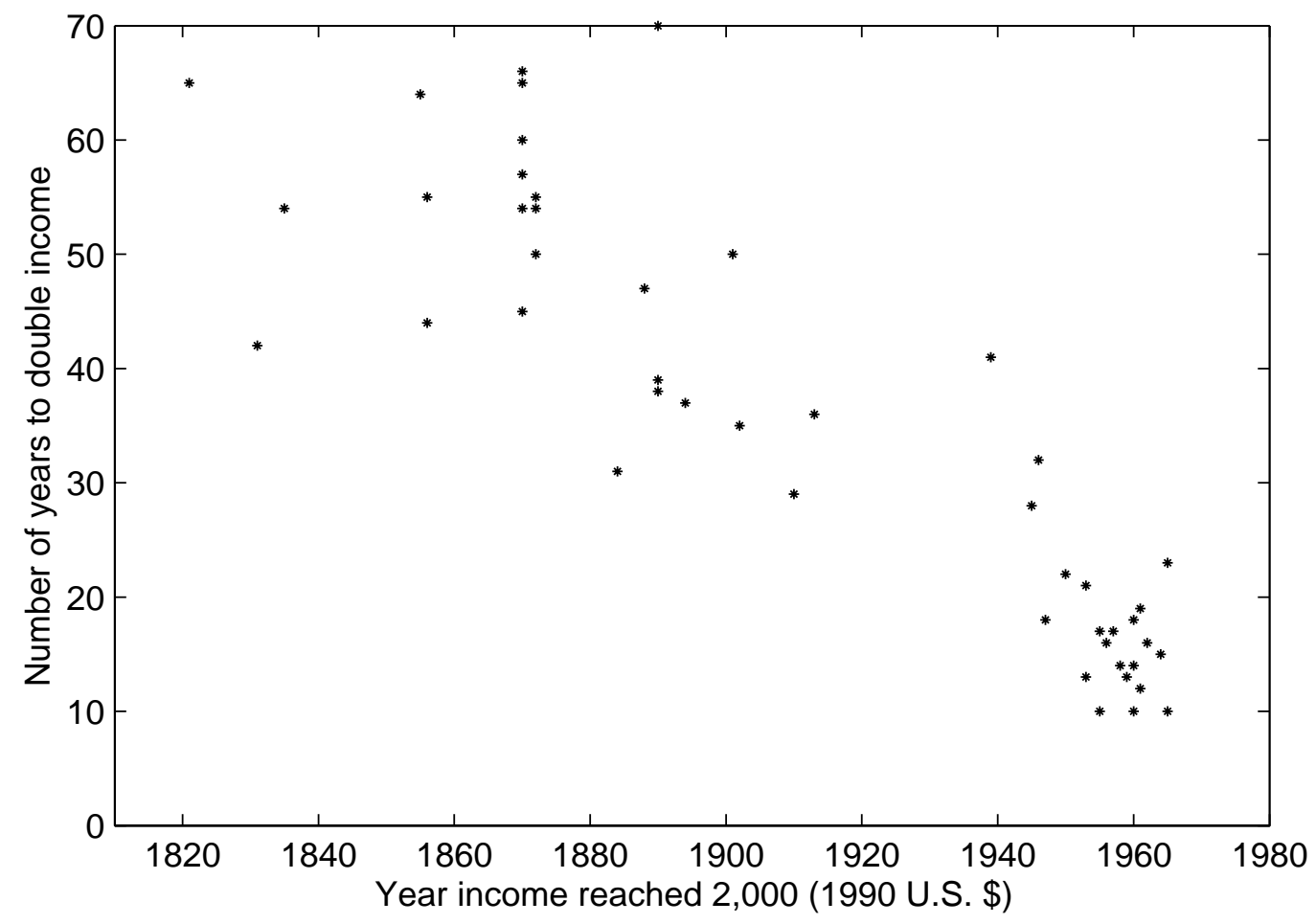

FIG. 1. Growth patterns from Parente and Prescott

\section{Introduction}

In Barriers to Riches, Parente and Prescott observe that "Countries reaching a given level of income at a later date typically double that level in a shorter time " To support this conclusion they present Figure 1

Figure 1 shows the year in which a country reached the per capita income level of 2,000 of 1990 U.S. dollars and the number of years it took that country to double its per capita income to 4,000 of 1990 U.S. dollars. Clearly the late starters grew much faster than the early starters did. This is more than convergence in the traditional sense. This graph is not only saying that poor countries grow faster than rich countries do. There is some kind of advantage that the late starters or followers have that early 
starters or leaders do not.

In this paper I present a simple model that generates the behavior shown in Figure 1. The model is a growth model with human capital accumulation and an externality in the human capital production function. This externality depends on the level of human capital of the most advance country. The model is similar to the model in Lucas(1993) with some differences. On the technology side model has no physical capital, and the external effect comes from the highest human capital level in the world. In Lucas' model is the average human capital level of the world that produces an external effect. I also introduce a utility maximization agent that optimally chooses the fraction of time that he allocates to invest in human capital. My model is similar to Parente and Prescott (1994) and Tamura (1996) in the sense that they also analyze the behavior of a maximizing agent that faces an external effect on the human capital production technology. In Section 2 I present the model and solve it analytically, in Section 3 I present a numerical example. Section 4 concludes the paper.

\section{The Model}

All countries are identical in preferences and technology. They only differ in their initial levels of human capital. Each country is in autarchy. There are no international markets for human capital or consumption goods. The only interaction among them comes from a learning externality. Each country is represented by a infinitely lived dynasty with preferences

$$
\int_{0}^{\infty} e^{-\rho t} U(c(t)) d t
$$

I assume that $U(c)$ takes the form

$$
U(c)=\frac{c^{1-\sigma}}{1-\sigma}
$$

Each country is endowed with one unit of time per period and they can use it 
to produce the consumption good $c(t)$ or to accumulate human capital $h(t)$. The fraction of time spent in the production of the consumption good is $u(t)$ There is an externality in the human capital sector. The level of human capital from the worlds most advance economy, $H(t)$, enters into the human capital accumulation function of all countries. Specifically, I assume

$$
c(t)=u(t) h(t)
$$

and

$$
\dot{h}(t)=\delta h^{1-\theta}(t) H^{\theta}(t)[1-u(t)] .
$$

All other countries have no effect on the human capital of the leader, but I also assume that all individuals in the leading economy are also affected by this external effect, and take $H(t)$ as given as well. One way to interpret this is that the leading country is composed of many identical households and that each household is too small to affect the aggregate human capital by its own actions.

The human capital of the leader is assumed to grow at a constant rate $\alpha$. Later, this guess will be verified and $\alpha$ will be viewed as an endogenous variable to be determined by the model. This implies that

$$
\dot{H}(t)=\alpha H(t),
$$

or alternatively

$$
H(t)=H(0) e^{\alpha t} .
$$

With this specification of preferences and technology I have enough information to set up and solve the model. Define

$$
x(t) \equiv \frac{h(t)}{H(t)} .
$$

so that (1) and (4)can be written as

$$
\frac{H^{1-\sigma}(0)}{1-\sigma} \int_{0}^{\infty} e^{-[\rho-\alpha(1-\sigma)] t}[u(t) x(t)]^{1-\sigma} d t
$$


and

$$
\dot{x}(t)=\delta x^{1-\theta}(t)[1-u(t)]-\alpha x(t)
$$

The problem faced by each country is to choose the function $u(t)$ to maximize $(5)$ subject to (6) given $x(0)$

From now on I assume that $\rho-\alpha(1-\sigma)>0$, so that the agent's utility is finite under all feasible plans, and $\delta>\rho$ so that the steady state growth rate is positive. Define the current value Hamiltonian $M$ as

$$
M \equiv \frac{(u x)^{1-\sigma}}{1-\sigma}+\psi\left[\delta x^{1-\theta}(1-u)-\alpha x\right]
$$

The maximum of $M$ can be found by setting

$$
\frac{\partial M}{\partial u}=0
$$

The rates of change of the co-state variable $\psi$, and the state variable $x$ are given by

$$
\dot{\psi}=[\rho-\alpha(1-\sigma)] \psi-\frac{\partial M}{\partial x}
$$

and

$$
\frac{\partial M}{\partial \psi}=\dot{x}
$$

The transversality condition for this problem is

$$
\lim _{t \rightarrow \infty} e^{-[\rho-\alpha(1-\sigma)] t} \psi(t) x(t)=0 .
$$

From these equations I obtain

$$
\begin{gathered}
u^{-\sigma}=\delta \psi x^{\sigma-\theta}, \\
\frac{\dot{\psi}}{\psi}=\rho+\alpha \sigma-(1-\theta+\theta u) \delta x^{-\theta},
\end{gathered}
$$

and

$$
\frac{\dot{x}}{x}=\delta x^{-\theta}(1-u)-\alpha .
$$


These equations describe both the behavior of the leading country and the followers. For the leader $h(t)=H(t)$ for all $t$ so $x(t)=1$ and $\dot{x} / x=0$. Then

$$
\begin{gathered}
u^{-\sigma}=\delta \psi, \\
\frac{\dot{\psi}}{\psi}=\rho+\alpha \sigma-(1-\theta+\theta u) \delta,
\end{gathered}
$$

and

$$
\delta(1-u)=\alpha
$$

Since $\alpha$ is constant, this implies that, for the leader, $u(t)$ is also constant, then from (10) $\psi=\frac{u^{-\sigma}}{\delta}$ is also constant, so $\dot{\psi} / \psi=0$. Using equation (12) together with equation (11) set to 0 I can solve for $\alpha$ and $u$. The complete solution to the leader's problem is

$$
\begin{gathered}
\alpha=\frac{\delta-\rho}{\sigma+\theta}, \\
u^{*}(t)=\frac{\rho+\delta(1-\sigma-\theta)}{(\sigma+\theta) \delta}, \\
c^{*}(t)=h^{*}(0)\left(\frac{\rho+\delta(1-\sigma-\theta)}{(\sigma+\theta) \delta}\right) e^{\left(\frac{\delta-\rho}{\sigma+\theta}\right) t},
\end{gathered}
$$

and

$$
h^{*}(t)=H(t)=h^{*}(0) e^{\left(\frac{\delta-\rho}{\sigma+\theta}\right) t} .
$$

The follower's behavior is also given by $(7)-(9)$. Differentiate (7) with respect to time, and use that result with (9) in (8) to eliminate the shadow price $\psi$. So (7) and (9) can be written just as functions of $u$ and $x$

$$
\begin{aligned}
\frac{\dot{u}}{u} & =-\frac{\rho+\alpha \theta-(1+\sigma u-\sigma) \delta x^{-\theta}}{\sigma} \\
\frac{\dot{x}}{x} & =\delta x^{-\theta}(1-u)-\alpha
\end{aligned}
$$

To find a steady state for the follower set $\dot{u} / u=\dot{x} / x=0$. Then $\dot{x}=0$ implies that

$$
u=1-\frac{\alpha}{\delta} x^{\theta}
$$


and $\dot{u}=0$ implies that

$$
u=\frac{(\rho+\alpha \theta)}{\delta \sigma} x^{\theta}+\frac{\sigma-1}{\sigma} .
$$

These two curves are plotted in Figure 2. A steady state is the intersection of this two curves. The intersection of this two curves when $\alpha=(\delta-\rho) /(1-\theta+\sigma)$ is

$$
x^{S S}=1
$$

and

$$
u^{S S}=\frac{\rho+\delta(1-\sigma-\theta)}{(\sigma+\theta) \delta}
$$

This are the same steady state values are the same than the ones from the leader.

Figure 2 plots the phase diagram for the system of differential equations represented by (13) and (14).

To analyze the stability of the steady state take a log-linear approximation of (13) and (14) around the steady state

$$
\left[\begin{array}{c}
\frac{d \log u}{d t} \\
\frac{d \log x}{d t}
\end{array}\right]=J \times\left[\begin{array}{c}
\log \left(\frac{u}{u^{S S}}\right) \\
\log (x)
\end{array}\right]
$$

The characteristic matrix $J$ is defined as

$$
J=\left[\begin{array}{cc}
\delta u^{S S} & -\frac{\left(1+\sigma u^{S S}-\sigma\right)}{\sigma} \delta \theta \\
-\delta u^{S S} & -\delta \theta\left(1-u^{S S}\right)
\end{array}\right]
$$

with

$$
\operatorname{det}(J)=-\frac{\delta^{2} u^{S S} \theta}{\sigma}<0
$$

This implies that there is saddle path stability, i.e. the eigenvalues of $J$ have opposite sign. So, locally, there is only one path or stable arm that converges to the steady state solution. I can solve the follower's problem with initial condition $x(0)$ by choosing the value of $u(0)$ such that the follower moves along the saddle path. Choosing an initial condition for $u(0)$ above the saddle path will make $u(t)$ grow 


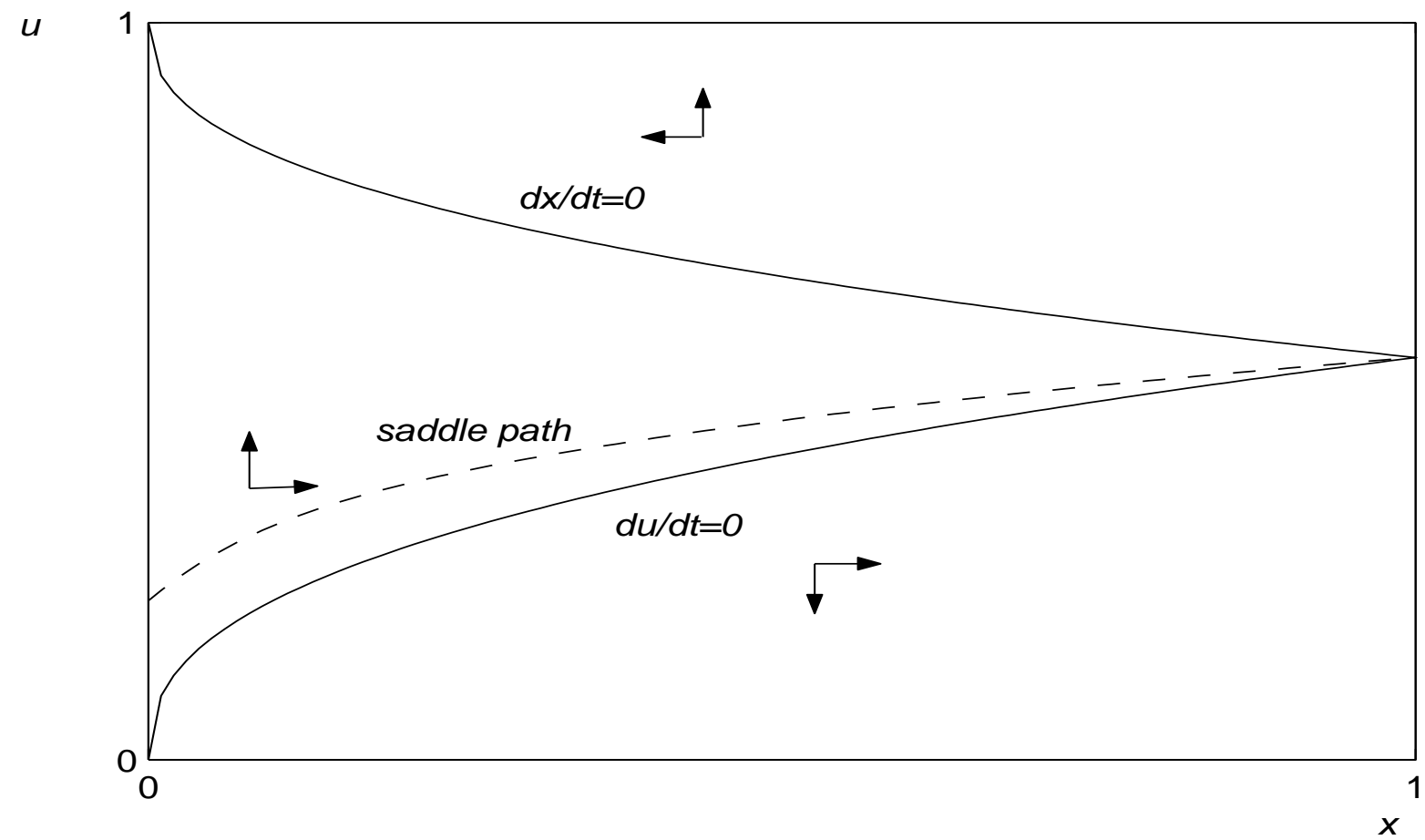

FIg. 2. Phase Diagram 
until it eventually becomes constant at a level of one. When $u(t)=1$ then $\psi(t) x(t)$ grows at the rate $\rho-\alpha(1-\sigma)$ and the transversality condition is violated. If instead we choose an initial condition below the saddle path then $x(t)$ will grow too fast and in finite time the country will become the world leader $x(t)=1$, and since $u(t)<u^{s s}$ at that given date, this implies that $u(t)$ must give a discrete jump, violating the first order condition for $u$.

This model has the property that for all $x(0)>0$ both $u(t)$ and $x(t)$ converge to the leader's values, if all the parameters of the model are the same. This implies that there is convergence in growth rates and output. Output convergence is defined as the convergence of the output ratio to one as time goes to infinity.

\section{A Numerical Example}

In this section I test if the model can generate the kind of behavior depicted in Figure 1. I assume that $\sigma=1$ and $\rho=0.02$. I simulate the model for $\theta=0.3, \theta=0.1$ and $\theta=0$ (this corresponds to the case with no learning externality), $\delta$ is chosen such that the long run growth rate $\alpha$ is the same in the three cases. I set $\alpha$ to 0.016 to match the growth rate of the US in the $1820-1990$ period $^{1}$. With the numerical solutions I can calculate how long it takes a country, that reached an income level of $\$ 2000 t$ years after the leader did, to double its income. The results are compared to the actual data in Figure 3. To match the model to the data I need to make an assumption about which country is the leader, so I assume that the US is the world leader.

The model captures the idea that late starters grow faster, but it fails to fit some aspects data. If I try to fit the data generated by the model to the linear trend in the Parente and Prescott data then the model would predict a growth rate for the leader (and long run growth rate for all the countries) of 1\%, which is inconsistent

\footnotetext{
${ }^{1}$ Data from Maddison (1995)
} 


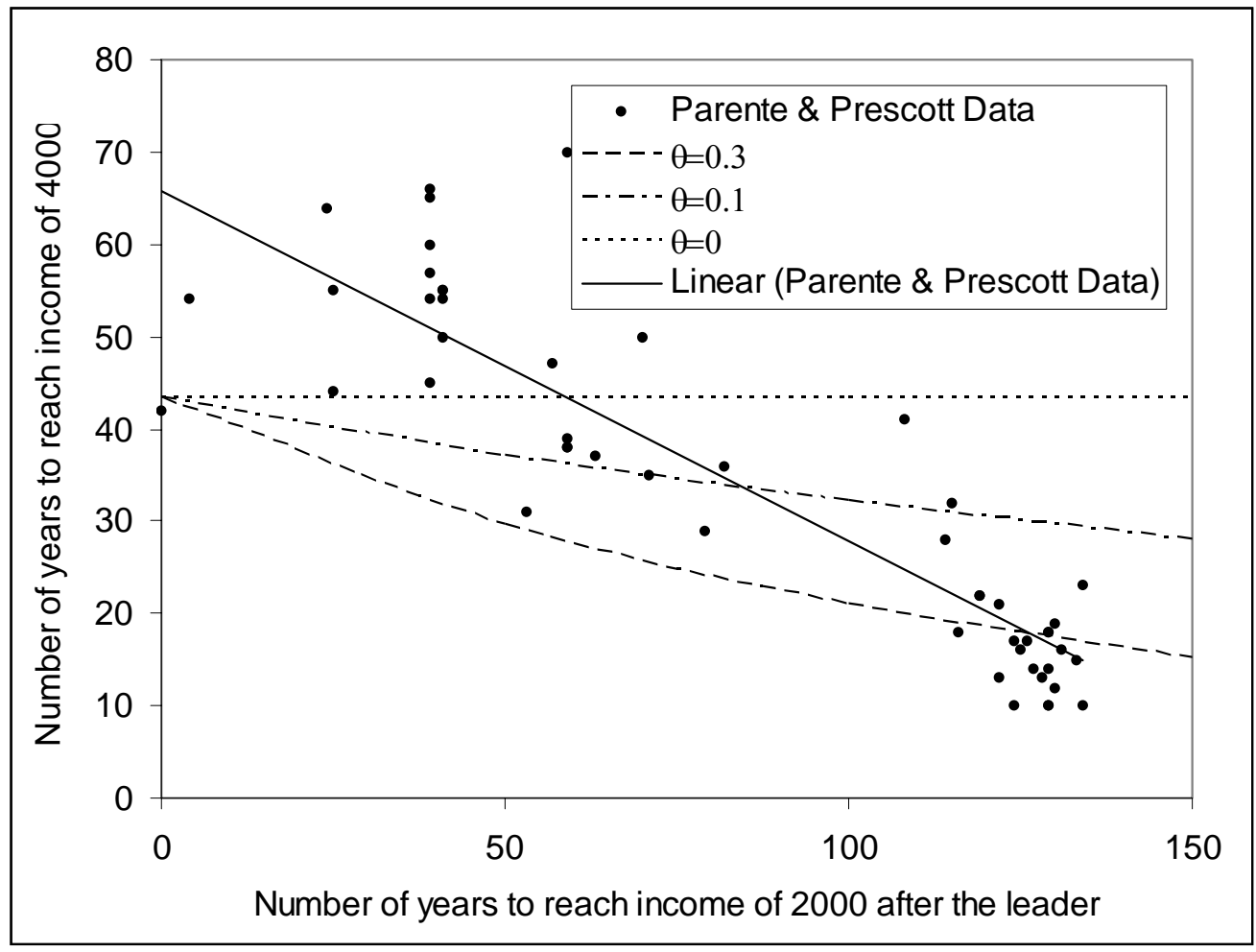

FIG. 3. Results of the simulations 
with the growth rate we observe for the US. If I calibrate the model to the have the observe growth rate of the US then the model predicts that no other country could have taken longer than 43 years to grow from an income level of 2000 to one of 4000 . Clearly the data shows otherwise. Countries that reached and income level of 2000 on the second half of the nineteenth century experienced growth rates much lower than those predicted by the model.

\section{Conclusions}

The model presented in this paper has the property that it can explain the behavior of a country like the US that has been growing steadily for almost 200 years, and the more recent miracles of economic growth, like South Korea and Taiwan, that had yearly growth rates of over $6 \%$ for over a decade. Still it fails to explain some other aspects of the data. These failures lead to new questions and future research.

The key factor of the model are learning externalities. Countries can take advantage of the human capital of the leader to increase their own. What determines this externalities is an important question to answer, since the answer will help many developing economies to make better policies. Lucas (1993) discusses what are the

possible sources of externalities in human capital accumulation. He comes to the conclusion that models in which the leaders human capital is a public good that is available to all countries have (like my model) the unpleasant characteristic that all countries will tend to grow. There is no room in this types of models for countries like Philippine and Korea, that were very similar in 1960, but experienced very different growth paths there after.

Learning externalities of the type proposed in this paper are not so difficult to imagine in the real world, but still they are not the only possibility. 


\section{REFERENCES}

[1] Barro, Robert J. and Sala-i-Martin Xavier. Economic Growth. MIT Press (1999)

[2] Chiang, Alpha C. Elements of Dynamic Optimization Waveland Press (2000)

[3] Lucas, Robert E. Jr. 1988 "On the Mechanics of Economic Development?" Journal of Monetary Economics, 22: 3-42.

[4] Lucas, Robert E. Jr. 1993. "Making a Miracle." Econometrica, 61: 251-272.

[5] Maddison, Angus. Dynamic Forces in Capitalist Development, A Long Run Comparative View. Oxford University Press (1991)

[6] Maddison Angus. Monitoring the World Economy: 1820-1992. Development Centre Studies (1995)

[7] Parente, Stephen L. and Prescott Edward C. Barriers to Riches. MIT Press (2000) 